\title{
Low-temperature transport in Heisenberg chains
}

\author{
J.V. Alvarez and Claudius Gros \\ Fakultät 7, Theoretische Physik, University of the Saarland, 66041 Saarbrücken, Germany.
}

\begin{abstract}
A technique to determine accurately transport properties of integrable and non-integrable quantum-spin chains at finite temperatures by Quantum Monte-Carlo is presented. The reduction of the Drude weight by interactions in the integrable gapless regime is evaluated. Evidence for the absence of a Drude weight in the gapless regime of a non-integrable system with longer-ranged interactions is presented. We estimate the effect of the non-integrability on the transport properties and compare with recent experiments on one-dimensional quantum-spin chains.
\end{abstract}

PACS numbers: 75.30.Gw, 75.10.Jm, 78.30.-j

Introduction - During the last few years several families of materials containing well characterized quasi onedimensional spin-1/2 structures have been synthesized. The charge-transfer gap is in many cases large and the spin excitations contribute significantly to the thermal and magnetization transport at low temperatures. For example, ${ }^{63} \mathrm{Cu}$ NMR studiest in $\mathrm{Sr}_{2} \mathrm{CuO}_{3}$ have measured a spin diffusion coefficient (equivalent to diffusive magnetization transport) several orders of magnitude larger than the value for conventional diffusive systems, and thermal transport measurements in $\mathrm{Sr}_{2} \mathrm{CuO}_{3}$ and $\mathrm{SrCuO}_{2}$ indicate 2 quasi-ballistic transport with a mean-free path of several thousands of $\stackrel{\AA}{A}$.

These unusual results have been related to the peculiar physics of one-dimensional quantum chains. It is known that the spin transport in the XXZ chain

$$
H^{(x x z)}=\sum_{i}\left[\frac{J_{x x}}{2}\left(S_{i}^{+} S_{i+1}^{-}+S_{i}^{-} S_{i+1}^{+}\right)+J_{z} S_{i}^{z} S_{i+1}^{z}\right]
$$

is not diffusive, even for $T \gg J_{x} 3$. A connection between integrability and transport 50 enlights the situation. In a generic integrable model, like $H^{(x x z)}$, the spin current is not conserved, but it has a non-vanishing 'component' with respect to the projection onto a conserved operator. As a consequence, the current-current correlation functions do not decay to zero for large times.

The hamiltonian appropriate for real compounds like $\mathrm{Sr}_{2} \mathrm{CuO}_{3}$ and $\mathrm{SrCuO}_{2}$ correspond to $H^{(x x z)}$ only in first approximation. A crucial question is therefore: How do small deviations from integrability change the picture described above? Extending an earlier analysis by Giamarchi10, Rosch and Andrei concluded recently within a memory-matrix approach 11 , that deviations from integrability lead to an exponentially large conductivity in Hubbard-like models away from commensurability.

Despite the on-going effort devoted to this problem, the fundamental difference in between integrable and non-integrable-models have not yet shown up in QMCsimulations 12 13, presumable due to the demanding numerical requirements. In this Letter we develop the techniques and tools for the data-analysis necessary for extracting the Drude weight for integrable and the lifetime, mean-free path and the diffusion constant for nonintegrable quantum-spin models from correlation functions obtained by QMC simulations using the Loop Algorithm14. We present extensive comparison with exact results obtained by Bethe-Ansatz and compare for a non-integrable system with recent experiments on $\mathrm{Sr}_{2} \mathrm{CuO}_{3}$.

Drude weight - QMC-simulations yield in general correlations functions on the imaginary-time axis. We therefore consider the Kubo formula for the dynamical conductivity $\sigma\left(\omega_{n}\right)=\lim _{q \rightarrow 0} \sigma\left(q, \omega_{n}\right)$

$$
\sigma\left(q, \omega_{n}\right)=\frac{-\langle K\rangle-\Lambda\left(q, \omega_{n}\right)}{\omega_{n}} \equiv \frac{D\left(q, \omega_{n}\right)}{\omega_{n}}
$$

on the imaginary axis, where $\langle K\rangle$, for the models we will consider, is the expectation value of the kinetic energy per site and where $\Lambda$ is the current-current correlation as a function of the Matsubara frequency,

$$
\Lambda\left(q, \omega_{n}\right)=\frac{1}{L} \int_{0}^{\beta} e^{i \omega_{n} \tau}\left\langle j^{z}(q, \tau) j^{z}(-q, 0)\right\rangle d \tau .
$$

Eq. (11) leads via $\sigma(\omega)=\pi D(T) \delta(\omega)+\sigma_{\text {reg }}(\omega)$ to the conventional relation 12 between the current-current correlation function and the Drude weight (we are borrowing the terminology of electrical responses) $D(T)=$ $\lim _{\omega_{n \rightarrow 0}} \lim _{q \rightarrow 0} D\left(q, \omega_{n}\right)$

$$
D(T)=-\langle K\rangle-\Lambda\left(q \rightarrow 0, \omega_{n} \rightarrow 0\right) .
$$

Note that $\Lambda(q, \omega+i \delta)$ is analytic in the upper half of the complex $\omega$-plane and the extrapolation along the imaginary axis can be reliably performed at low temperatures, when many Matsubara-frequencies $\omega_{n}=2 \pi T n$ are available close to $\omega=0$ for the extrapolation 13 .

The continuity equation $\frac{\partial}{\partial t} S_{l}^{z}(t)+\frac{\partial}{\partial x} j_{l}^{z}(t)=0$ leads to the expression

$$
j_{l}^{z}=-i J_{x x}\left(S_{l}^{+} S_{l+1}^{-}-S_{l}^{-} S_{l+1}^{+}\right)
$$

for the current operator $j_{l}(t)$ in the XXZ-model. In Fourier space, the continuity equation takes the form

$$
\frac{d}{d \tau} S_{q}^{z}(\tau)=\left[H, S_{q}^{z}\right]=i\left(1-e^{i q}\right) j_{q}^{z}
$$

$\Lambda\left(q, \omega_{n}\right)$ is a non-diagonal four-site operator. In principle non-diagonal operators can be computed using the loop 
algorithm 15 but, as it was discussed recently 16 , the algorithm to compute two-site correlation functions is more efficient than the algorithms that compute operators involving three or more sites. To obtain high quality data we measure directly the dynamical susceptibility in imaginary time

$$
S\left(q, \omega_{n}\right)=\frac{1}{L} \int_{0}^{\beta} e^{i \omega_{n} \tau}\left\langle S_{q}^{z}(\tau) S_{-q}^{z}(0)\right\rangle,
$$

a simple diagonal two-site correlation function. The Drude peak is computed by using Eq. (3) and the relation

$$
\omega_{n}^{2} S\left(q, \omega_{n}\right)=4 \sin ^{2}(q / 2) D\left(q, \omega_{n}\right) .
$$

This relation can be derived by a two-fold partial integration of the right-hand side of Eq. (6) with respect to $\tau$, which leads to

$$
S\left(q, \omega_{n}\right)=\frac{-1}{\omega_{n}^{2}}\left\langle\left[\left[H, S_{q}^{z}\right], S_{-q}^{z}\right]\right\rangle-\frac{4 \sin ^{2}(q / 2)}{\omega_{n}^{2}} \Lambda\left(q, \omega_{n}\right),
$$

where we have used Eq. (5) for the imaginary-time derivatives of $S_{q}^{z}$ and $S_{-q}^{z}$ and definition (2). Evaluation of the double commutator $\left[\left[H, S_{q}^{z}\right], S_{-q}^{z}\right]$, which is the boundary term from the partial integration, then leads to Eq. (7). Note, that this double commutator occurs here for the Matsubara correlation functions and does not occur for a related real-frequency correlation functions.

Data-Analysis - At low temperatures and frequencies, the scaling of $D\left(q, \omega_{n}\right)$ can be obtained simply invoking the conformal symmetry of the model emerging in the gapless regime $J_{z}<J_{x x} . S\left(q, \omega_{n}\right)$ at small q takes then form $S\left(q, \omega_{n}\right)=D_{1}(T) q^{2} /\left((c q)^{2}+\omega_{n}^{2}\right)$. This expression and Eq. (7) suggest the form

$$
D\left(q, \omega_{n}\right)=\frac{D_{1}(T) \omega_{n}^{2}}{\Delta^{2}(q)+\omega_{n}^{2}} .
$$

Alternatively, Eq. (8) can be viewed as the first term of the exact representation for $D\left(q, \omega_{n}\right)$ containing an infinite-number of terms 13 .

The XXZ-model maps to an interacting 1D spinless fermionic system at half filling. For the noninteracting case (the XX chain) we can compute exactly $D\left(q, \omega_{n}\right)$ and we obtain $D(0)=J_{x x} / \pi$, and $\Delta(q)=J_{x x} \sin (q) \sim J_{x x} q$. For $J_{z}<J_{x x}$ the Umklapp term remains marginally irrelevant and one expects Luttinger Liquid like correlation functions 10 like Eq. (8).

In the ideal Luttinger Liquid the bosonic excitations are arbitrarily well defined at low temperatures and low frequencies. The conductivity is necessarily infinite in that case, and ansatz (8), being invariant under time reversal reflects that property. Nevertheless, we are also interested in the study of more general situations in which the bosons can decay, become quasiparticles, and memory effects can be taken into account. To this end we use the formalism of the memory matrices which has been successfully applied to study howene-dimensional electron liquids can gain a resistivity 10.11.

This is achieved by taking the following fitting function for $D\left(q, \omega_{n}>0\right)$ :

$$
D\left(q, \omega_{n}\right)=\sum_{j=1}^{2} \frac{D_{j}(q) \omega_{n}^{2}}{\Delta_{j}^{2}(q)+2 \gamma_{j}(q) \omega_{n}+\omega_{n}^{2}}
$$

$D\left(q, \omega_{n}\right)$ is analytic in the upper complex-plane for $\gamma_{j}(q) \geq 0$. For $\Delta_{i}(0)=\lim _{q \rightarrow 0} \Delta_{i}(q)$ we find either a

(i) gapless regime: $\Delta_{1}(0)=0$ and $\Delta_{2}(0)>0$, or a

(i) gap-full regime: $\Delta_{1}(0)>0$ and $\Delta_{2}(0)>\Delta_{1}(0)$.

The first term in Eq. (9) dominates the low-frequency behavior in both cases and we have set generally $\gamma_{2} \equiv 0$ in order to keep the number of parameters to a minimum.

In the gapless regime the optical conductivity (1) takes for small frequencies the Drude form

$$
\operatorname{Re} \sigma(\omega)=\frac{2 D_{1}(0) \gamma_{1}(0)}{\omega^{2}+4 \gamma_{1}^{2}(0)} \equiv \frac{\sigma_{0}}{1+(\omega \tau)^{2}}
$$

where we introduced the DC-conductivity $\sigma_{0}=$ $D_{1}(0) /\left(2 \gamma_{1}(0)\right)$ and the quasi-particle lifetime $\tau=$ $\left(2 \gamma_{1}(0)\right)^{-1}$. For $\tau \rightarrow \infty$ Eq. (10) reduces to $\operatorname{Re} \sigma(\omega)=$ $\pi D_{1}(0) \delta(\omega)$. At high frequencies

$$
\lim _{\omega_{n} \rightarrow \infty} D\left(0, \omega_{n}\right)=-\langle K\rangle \equiv D_{1}(0)+D_{2}(0)
$$

and a finite $D_{2}(0)$ results in a reduction of the Drude weight $D(T)$ with respect to the kinetic energy, see Eq. (3). A finite $D_{2}(0)$ measures therefore the amount of decay experienced by the total current due to the interactions. We note that the Ansatz Eq. (9) for $D\left(q, \omega_{r}\right)$ together with Eq. (11), is consistent with the f-sum-rule 17 $\int_{0}^{\infty} \operatorname{Re} \sigma(\omega)=-\frac{\pi}{2}\langle K\rangle$ for the optical conductivity.

Ballistic transport - In Fig. 11 we show the values for $D_{1}(q)$ and $\Delta_{1}(q)$ for the $\mathrm{XX}$ chain for different system sizes as obtained by QMd18, $D_{2}(q)$ and $\gamma_{1}(q)$ optimize to zero for this model. The different system sizes collapse at the smallest momenta and in this way the thermodynamic limit and the $q \rightarrow 0$ limit are performed simultaneously. Our prescription to extract $D_{1}(0)$ as the limiting value $D_{1}(0)=\lim _{q \rightarrow 0} D_{t}(q)$ is more involved than previous $q \equiv 0$ calculations 13 . but we believe it to be necessary in order to avoid substantial finite-size effects due to the fact that $D\left(q \rightarrow 0, \omega_{n}\right)$ becomes a step-function at $\omega_{n}=0$ in the thermodynamic limit, compare Eq. (8).

For finite interaction $J_{z}$ we find in the gapless regime $J_{z} \leq J_{x x}$, that $\Delta_{1}(q) \approx c\left(J_{z}\right) q$ where $c\left(J_{z}\right)$ is the velocity

$$
c\left(J_{z}\right)=\frac{\pi}{2} \frac{\sqrt{J_{x x}^{2}-J_{z}^{2}}}{\operatorname{arcos}\left(J_{z} / J_{x x}\right)}
$$

of the des Cloiseaux-Pearson spectrum 19. In Fig. 2 2 we compare the measured value of $c\left(J_{z}\right)$ with the Bethe Ansatz result (12) for $J_{z} / J_{x y}=1 / 2,1 . \Delta_{1}(q)$ is fitted well in the gapped phase by $\varepsilon(q)=\sqrt{\Delta_{0}^{2}+(c q)^{2}}$. We 
find $\Delta_{0}=0.191 J_{x x}$, which is close to twice the onemagnon gap of $0.091 J_{x x} 19$.

The damping $\gamma_{1}(q)$ is vanishing small for $J_{z}<J_{x x}$ and acquires a finite value in the gapped phase which can be fitted phenomenological by the relation $\gamma_{1}(q) \Delta_{1}(q) \approx$ const., independent of $q$.

In Fig. 3 (inset) we present values obtained by QMC for the Drude weight in the gapless regime, $J_{z}<J_{x x}$ at $T=0.004 J_{x x}$. We find good agreement with the $T=0$ Bethe Ansatz resulte

$$
D(0)=\frac{\nu^{2}}{4 \pi} \frac{\sin (\pi / \nu)}{\nu-1}, \quad J_{z}=J_{x x} \cos (\pi / \nu)
$$

specially at the smaller values of $J_{z}$. In this region the correlation function is expected to satisfy more accurately the scaling law (8). The $S\left(q, \omega_{n}\right)$ in the isotropic Heisenberg model does not follow strictly the Luttinger Liquid form. It presents multiplicative logarithmic corrections, that should be observed also for values of $J_{z}$ slightly smaller than $J_{x x}$ when finite temperatures and system sizes impose a cut-offfor the RG equation of the Umklapp coupling constant 10 .

We study now the behavior of the Drude weight at finite temperatures for models free from strong multiplicative corrections. The main conclusion of Zotos 21 is the fast decay of the Drude weight when the temperature increases, $D(T)-D(0) \sim-\kappa T^{\frac{2}{\nu-1}}$ where $\kappa$ is a constant and $\nu$ defined by Eq. (13). This rapid decrease with increasing temperature is consistent with exact diagonalization studies at hight temperatures 9 . Klümper et al. have found on the other hand22, with an alternative Bethe-ansatz approach, a functionally different behavior for $D(T)$, see Fig. A. For a numerical probe of $D(T)$ we focus on $\nu=6$ and consider several small temperatures. In Fig. (4) we show a comparison our data with the two available analytical results21.22. Our results agree with the temperature-dependence predicted by Klümper et al., suggesting in particular a finite Drude weight also at the isotropic pointe2.

Diffusive transport - We consider now a perturbation of $H^{(x x z)}$ that breaks the integrability but keeps the $z$-component of the magnetization conserved:

$$
H^{\prime}=J_{z}^{\prime} \sum_{i} S_{i}^{z} S_{i+3}^{z}
$$

The expression (4) for the spin current remains valid. For this model, $H=H^{(x x z)}+H^{\prime}$, we predict a transition to a gapped phase around $J_{z}^{\prime} \cong 0.3 J_{x x}$ (for $\left.\nu=6\right)$, see Fig. 3. We find the relaxation time $\tau=1 / 2 \gamma_{1}(0)=$ $\lim _{q \rightarrow 0} 1 / 2 \gamma_{1}(q)$ to be finite within the numerical accuracy (due to finite- $q$ and $\omega_{n}$ resolution), leading to a finite DC-conductivity in the gapless phase.

For $1 / \omega \gg \tau$ the optical conductivity takes (for small $\left.c q / \gamma_{1}(0)\right)$ the diffusion form

$$
\sigma(q, \omega)=\frac{\sigma_{0} \omega}{\omega+i D_{s} q^{2}}, \quad D_{s}=\frac{c^{2}}{2 \gamma_{1}(0)} \equiv c^{2} \tau .
$$

$D_{s}$ is the spin-diffusion constant. Eq. (15) is consistent with $D_{s}=c \lambda_{s}$, where $\lambda_{s}=c \tau$ is the mean free length.

We have evaluated $\sigma(T)$, in addition to the data presented in Fig. 3, for $\nu=6, J_{z}^{\prime}=0.3 J_{x x}$ and find $\sigma(T=$ $\left.0.004 J_{x x}\right)=13.6 \pm 0.9, \sigma\left(T=0.008 J_{x x}\right)=12.1 \pm 1.0$ and $\sigma\left(T=0.012 J_{x x}\right)=10.1 \pm 0.8$.

We now take $J_{x x}=2000 \mathrm{~K}$, which is appropriate for $\mathrm{Sr}_{2} \mathrm{CuO}_{3}$ and evaluate the transport coefficients for $J_{z}^{\prime}=0.3 J_{x x}, \nu=6, T=0.004 J_{x x}$. We find $\lambda \approx 88$ lattice constants and $D_{s} \approx 6 \times 10^{15} \mathrm{sec}^{-1}$. We do not expect this model to be directly relevant for $\mathrm{Sr}_{2} \mathrm{CuO}_{3}$. But if we ask ourselves now the question, whether the experimental results for $\lambda$ and $D_{s}$ being large (but finite) could be explained within a pure spin-model with a small deviation from integrability, we might expect $H=H^{(x x z)}+H^{\prime}$ to show the characteristic behavior of a non-integrable quantum-spin chain. If we now change the deviation from integrability (controlled by $J_{z}^{\prime}$ ) such that $\gamma_{1}(0)$ decreases by a factor of about ten, then both $\lambda$ and $D_{s}$ would increase by the same factor and would be consistent with the experimentally measured values 1 . 2 .

Discussion - We have shown that certain transport properties of quantum-spin chains can be evaluated directly from two-point correlations functions using a relation in between $S\left(q, \omega_{n}\right)$ and the the dyamical conductivity which we have derived. For the integrable chains we support the original suggestion by Zotos et al.5 of a finite Drude weight at finite temperatures and settle a recent dispute regarding the functional form of $D(T)$. In addition we presents results suggesting the absence of ballistic transport (i.e. zero Drude-weight) for a nonintegrable model, for which we are able to estimate magnitude of the DC-conductivity. We have shown that the experiments on quasi one-dimensional spin-compound 1 , 2 are, in principle, consistent with the notion that they probe directly non-integrability effects, though we cannot rule ouf at this point that disorder or the coupling to phonons 1 would lead to the observed finite values for the transport coefficients.

Acknowledgments - This work was supported by the DFG. We would like to thank A. Klümper and X. Zotos for giving us their numerical results and their valuable comments.We also want to thank A.Sologubenko and R. Valentí a careful reading and several suggestions.

${ }^{1}$ M. Takigawa, N. Motoyama, H. Eisaki and S Uchida, Phys. Rev. Lett. 76, 4612 (1996).

${ }^{2}$ A.V. Sologubenko, K. Gianno, E. Felder, H.R. Ott, A. Vietkine and A. Revcolevschi Phys. Rev. B 62, R6108(2000) and cond-mat/0103419.

${ }^{3}$ B.N. Narozhny, Phys. Rev. B 96, 3311 (1996).

${ }^{4}$ K. Fabricius, U. Löw and J. Stolze, Phys. Rev. B 55, 5833 (1997). 
${ }^{5}$ H. Castella, X. Zotos, and P. Prelovšek, Phys. Rev. Lett. 74, 972 (1995).

${ }^{6}$ X. Zotos and P. Prelovšek, Phys. Rev. B 53, 983 (1996).

${ }^{7}$ X. Zotos, F. Naef, and P. Prelovšek, Phys. Rev. B 55, 11029 (1997).

${ }^{8}$ F. Naef and X. Zotos, J. Physics, Cond. Matt. 10, L183 (1998).

${ }^{9}$ B.N. Narozhny, A.J. Millis and N. Andrei, Phys. Rev. B 58, R2921 (1998).

10 T. Giamarchi, Phys. Rev. B 46, 342 (1992).

11 A. Rosch and N. Andrei, Phys. Rev. Lett. 85, 1092 (2000).

12 D.J. Scalapino, S.R. White, and S. Zhang, Phys. Rev. B 47, 7995 (1993)

${ }^{13}$ S. Kirchner, H.G. Evertz and W. Hanke, Phys. Rev. B 59, 1825 (1999).

${ }^{14}$ H.G. Evertz, G. Lana and M. Marcu, Phys. Rev. Lett. 70, 875 (1993).

${ }^{15}$ R. Brower, S. Chandrasekaran and U.-J. Wiese, Physica A 261, 520 (1998).

${ }^{16}$ J. V. Alvarez and C. Gros. Eur. Phys. J. B, 15, 641 (2000)

${ }^{17}$ See, e.g. D. Baeriswyl, C. Gros and T.M. Rice, Phys. Rev. B 36, 839 (1987).

${ }^{18}$ We used discrete imaginary-time decomposition with typical $N_{T}=800-2000$ and on the avarage $610^{6}$ full MCupdates in the grand-canonical ensemble. Test-runs within a canonical ensemble were performed.

19 J. des Cloiseaux and M. Gaudin, J. Math. Phys. 7, 1384 (1966).

${ }^{20}$ B. S. Shastry and B. Shutherland, Phys. Rev. Lett. 65, 243 (1990).

${ }^{21}$ X. Zotos, Phys. Rev. Lett. 821764 (1999)

${ }^{22}$ A. Klümper, private communications and J. Benz, T. Fukui, A. Klümper and C. Scheeren (in preparation).

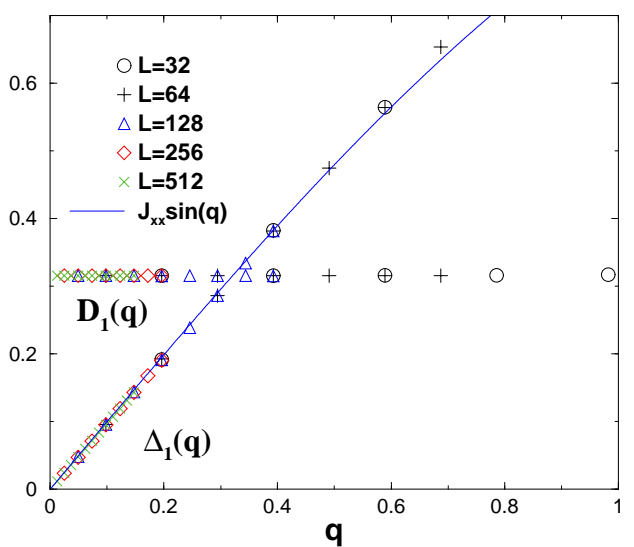

FIG. 1. The parameters $D_{1}(q)$ and $\Delta_{1}(q)$ from Eq. (9) as a function of momenta $q$, for the XX-model $\left(J_{z}=0\right)$ and various system sizes at $T=0.004 J_{x x}$. Statistical error bars are of the order of the system size 18 .

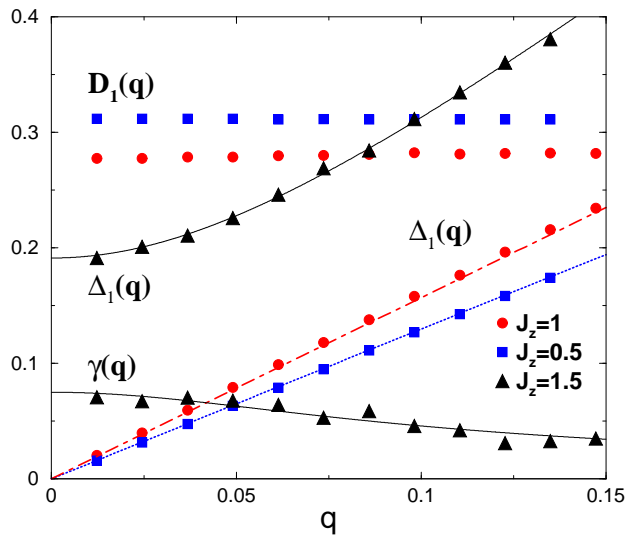

FIG. 2. $\quad D_{1}(q), \Delta_{1}(q)$ and $\gamma_{1}(q)$ from Eq. (9) as a function of momenta $q$ for the XXZ-model, $L=512$ and for various $J_{z}$ at $T=0.004 J_{x x} \cdot \gamma_{1}(q)$ is too small for $J_{z} \leq J_{x x}$ to show up on this scale. The lines are the Bethe Ansatz result (12) for the velocity $c\left(J_{z}\right)$ (no fit, for $\left.J_{z} \leq J_{x x}\right)$. For the discussion of the fit for $J_{z}=1.5 J_{x x}$ see the text.

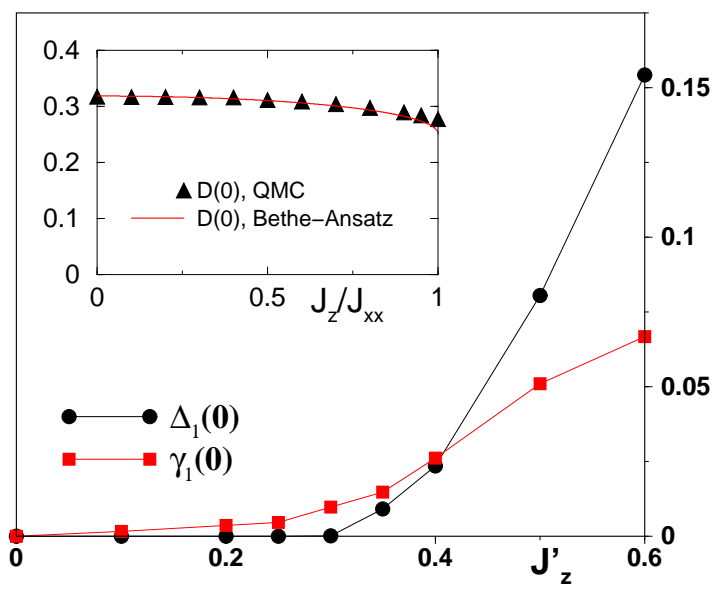

FIG. 3. For $L=512$ and $T=0.004 J_{x x}$ the QMC-results for the gap $\Delta_{1}(0)$ and the relaxation rate $\gamma_{1}(0)$ as a function of $J_{z}^{\prime}$ for $H^{(x x z)}+H^{\prime}$ with $J_{z}=J_{x x} \cos (\pi / 6)$. Inset: The QMC-results for the Drude weight for $J_{z}^{\prime}=0$ and $T=0.004 J_{x x}$ and $L=512$ in comparison with the Bethe-Ansatz result at $T=0$, Eq. (13). Note that $D(T)$ is smooth for low $\mathrm{T}$ (see Fig. 襾). 


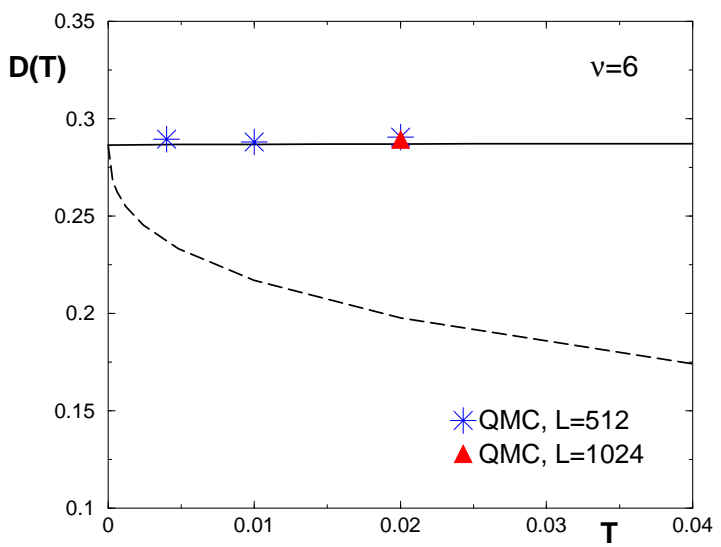

FIG. 4. QMC results for the Drude weight for $L=512,1024$ and $J_{z}=J_{x x} \cos (\pi / 6)$ as a function of temperature (in units of $J_{x x}$ ) in comparison with two (solid lines: Ref 22, dashed lines: Ref21) Bethe-Ansatz results. 